\title{
A Study on the Longitudinal and Lateral Errors of Air Vehicle Heading for Auto-landing
}

\author{
Ji Hee Park ${ }^{1 \dagger}$, Hong Sick Park', Chul Su Shin', Young-Wo Jo², Dong-Ho Shin ${ }^{2}$ \\ ${ }^{1}$ LIGNexl Co., Ltd, 100 Sinseong-dong, Yuseong-gu, Daejeon 305-804, Korea \\ ${ }^{2}$ Agency for Defense Development, 111 Sunam-dong, Yuseong-gu, Daejeon 305-152, Korea
}

\begin{abstract}
For the auto-landing operation of an air vehicle, the possibility of auto-landing operation should be first evaluated by testing the navigation performance through a flight test. In general, navigation performance is tested by analyzing north/east/down (NED) errors relative to reference equipment whose precision is about 8 10 times higher than that of a navigation system. However, to evaluate the auto-landing operation of an air vehicle, whether the air vehicle approaches a glide path aligned with the runway, within a specific error, needs to be examined rather than examining the north/east errors of the navigation system. Therefore, the longitudinal/lateral errors of air vehicle heading need to be analyzed. In this study, a method for analyzing the longitudinal/lateral errors of a navigation system was proposed as the navigation performance test method for evaluating the safety during the auto-landing of an air vehicle. Also, flight tests were performed six times, and the safety of auto-landing was examined by analyzing the performance using the proposed method.
\end{abstract}

Keywords: north/east error, longitudinal/lateral error, air vehicle, heading

\section{INTRODUCTION}

For the landing process of a fixed-wing air vehicle, the flight time is relatively short compared to that of cruising. However, as shown in Fig. 1, the accident rate during landing amounts to about $36 \%$, and it amounts to about $46 \%$ when the initial approach for landing is included (Boeing 2009). Also, as shown in Fig. 2, the sources of system failures for the unmanned aerial vehicle systems of Israel and the United States, which have the most abundant experience of unmanned aerial vehicle operation, indicated that flight control is one of the major causes of the unmanned aerial vehicle accidents (Office of Secretary of Defense 2005). Therefore, the stable flight and landing of an air vehicle should be secured, and the reliability should also be improved. Accordingly, research and development have been actively conducted on a number of automatic systems such as high-performance automatic flight control system, navigation system, and automatic landing system

Received Sep 30, 2013 Revised Oct 28, 2013 Accepted Oct 29, 2013 †Corresponding Author

E-mail: jihee.park@lignex1.com

Tel: +82-42-718-3553 Fax: +82-42-718-3470
(Shue \& Agarwal 1999, Noh \& Agarwal 2000). The automatic landing system of an air vehicle can be broadly divided into the control, navigation, and guidance subsystems. As the performance of each subsystem directly affects the safety and reliability of an air vehicle, it is very important to test the performance through a flight test. Especially, control and guidance subsystems perform the generation and guidance of the landing trajectory of an air vehicle using the navigation information of the navigation system. Thus, before performing auto-landing, navigation performance test should be performed though a flight test, to evaluate the possibility of auto-landing.

Navigation performance evaluation can be performed by analyzing north/east/down (NED) errors relative to a reference trajectory which has higher navigation accuracy than the navigation system of an air vehicle. The navigation accuracy of a reference trajectory needs to be about 8 10 times higher than that of the target equipment for performance evaluation (Sabatini \& Palmerini 2000). However, when an air vehicle enters auto-landing mode, the control and guidance subsystems should make the air vehicle approach a glide path that is aligned with the runway, for the safe landing of the air vehicle. Therefore, the longitudinal/lateral/ vertical (LLV) errors of air vehicle heading are more important than the NED errors. Also, when an air vehicle lands 


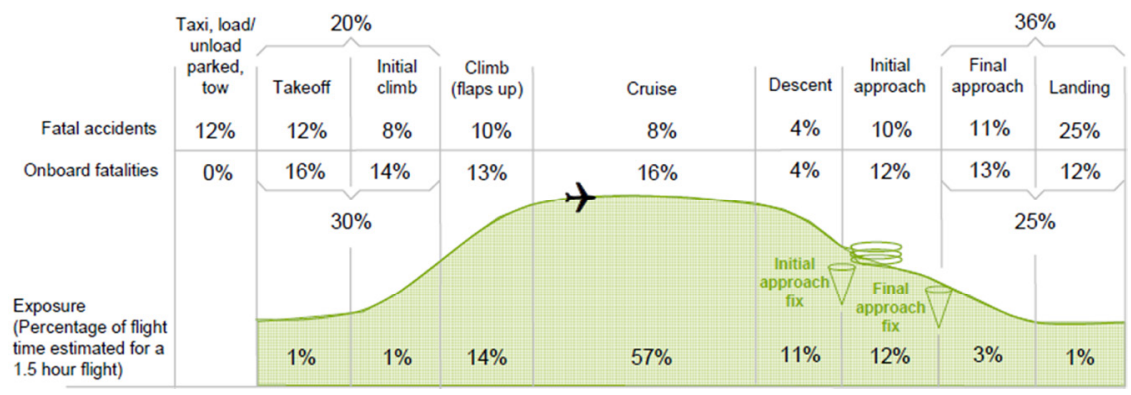

Fig. 1. Percentage of accidents/fatalities by the phase of flight.

on a runway, spaces are sufficiently secured in the heading direction, and thus the lateral error should be more strictly checked than the longitudinal error. However, as for the altitude information for auto-landing, an air vehicle uses a radar altimeter, which is a sensor that measures the distance between the sensor and the ground by transmitting radar signals and receiving the signals reflected from the ground. At low altitude, a radar altimeter has a precision of less than $1 \mathrm{~m}$ or several meters, and it is commonly used to monitor the distance to the ground during the takeoff and landing of an air vehicle. Therefore, in this paper, only horizontal errors will be discussed (Yoo et al. 2011, Yoon et al. 2013).

In this study, a method for analyzing the longitudinal/ lateral errors of a navigation system was proposed as the navigation performance test method for evaluating the safety during the auto-landing of a general air vehicle or an unmanned aerial vehicle. Also, flight tests were performed six times to compare the proposed method with the existing method using NED errors. Then the safety of auto-landing was examined based on the results of the analysis using the proposed method. The contents of this paper are as follows. In Chapter 2, the meaning of the longitudinal/ lateral/vertical frame (LLV-frame) of air vehicle heading was defined, and the difference between the LLV-frame and the body frame or navigation frame was explained. Then a method for transforming body errors or NED errors
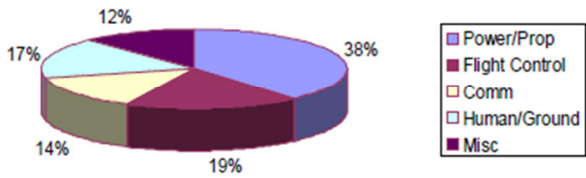

(a) U.S military UA fleet

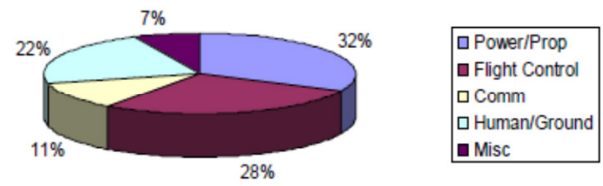

(b) Israeli Aircraft Industries (IAI) UA fleet

Fig. 2. Average sources of system failures for UAV. into LLV errors was proposed. In Chapter 3, the newly proposed method was applied to the performance analysis of navigation equipment following an actual flight test, and the results of the longitudinal/lateral error analysis were presented. In Chapter 4, conclusions were drawn.

\section{LONGITUDINAL/LATERAL FRAME OF AIR VEHICLE HEADING}

\subsection{Definition of the LLV-frame of Air Vehicle Heading}

For expressing the position, velocity, and attitude of a moving object, there are several kinds of coordinate systems, and an appropriate coordinate system is selected considering the simplification of navigation equations and the reduction of calculation as well as the requirements of a target navigation system. The representative coordinate systems that are typically used in an air vehicle include the body frame and the navigation frame (i.e., NED frame). As shown in Fig. 3, for the body frame, the origin is placed at the center of gravity of an air vehicle. Then the nose direction is defined as the $\mathrm{X}_{\mathrm{b}}$-axis, the right wing direction is defined as the $Y_{b}$-axis, and the downward direction is

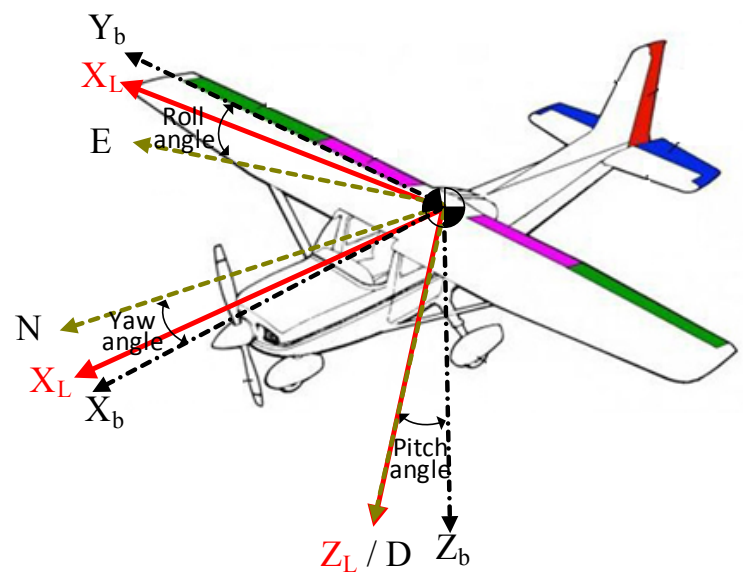

Fig. 3. LLV frame of air vehicle heading. 


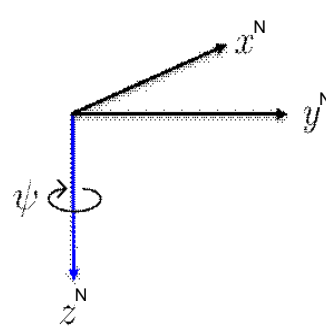

(a) Rotation about $\mathrm{z}^{\mathrm{N}}$ through angle $\Psi$

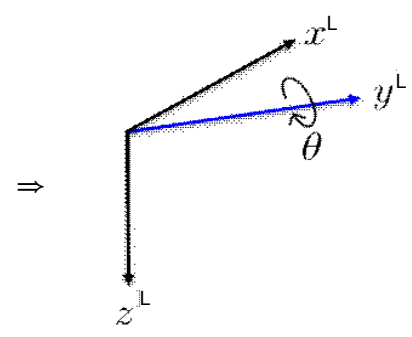

(b) Rotation about $y^{1}$ through angle $\theta$

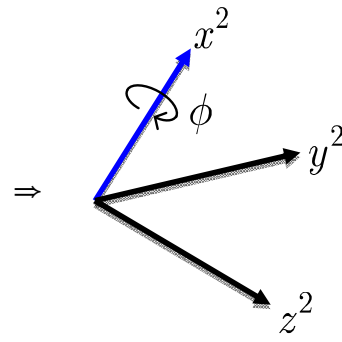

(c) Rotation about $\mathrm{x}^{2}$ through angle $\varphi$

Fig. 4. Rotation from the $n$-frame to the b-frame.

defined as the $\mathrm{Z}_{\mathrm{b}}$-axis. For the navigation frame, which is used for calculating the actual trajectory and position of an air vehicle, the origin is also placed at the center of gravity of an air vehicle. The $\mathrm{N}$ vector points to the north, the $\mathrm{E}$ vector points to the east, and the $\mathrm{D}$ vector points to the gravity vector direction (Farrell \& Barth 1998). As shown in Fig. 3, for the lateral/longitudinal/vertical frame (LLVframe: $\mathrm{X}_{\mathrm{L}}, \mathrm{Y}_{\mathrm{L}}, \mathrm{Z}_{\mathrm{L}}$ ) of air vehicle heading proposed in this study, the origin is placed at the center of gravity of an air vehicle. The longitudinal axis is defined as the air vehicle heading direction on a horizontal plane, the lateral axis is defined as the right direction of an air vehicle relative to the longitudinal axis, and the vertical axis is defined as the vertical downward direction of an air vehicle. The LLVframe can be matched with the body frame ( $b$-frame: $X_{b}, Y_{b}$, $\mathrm{Z}_{\mathrm{b}}$ ) and the navigation frame ( $\mathrm{n}$-frame: $\mathrm{N}, \mathrm{E}, \mathrm{D}$ ) by rotation about the same origin, and this rotation corresponds to the attitude of an air vehicle. In other words, the b-frame can be matched with the LLV-frame by the rotation of the roll and pitch angles of an air vehicle, and the n-frame can be matched with the LLV-frame by the rotation of the yaw angle. Fig. 4 shows the rotation of the Euler angles for transforming the $\mathrm{n}$-frame into the $\mathrm{b}$-frame. The LLV-frame is the same as the L-frame shown in Fig. $4 \mathrm{~b}$.

Based on the definitions of the coordinate systems, Fig. 5 shows the position that a navigation system outputs, relative to the actual position of an air vehicle $\left(R_{\text {ref }}\right)$ when the air vehicle has approached a glide path for landing. For the auto-landing of an air vehicle, whether the air vehicle is aligned with the runway should be examined, and thus the lateral/longitudinal errors of air vehicle heading need to be checked rather than the north/east errors.

\subsection{Coordinate Transformation Method from the $n$-frame or the b-frame to the LLV-frame}

In general, the coordinate transformation matrix for transforming the $\mathrm{n}$-frame into the $\mathrm{b}$-frame $\left(\mathrm{C}_{\mathrm{N}}^{\mathrm{B}}\right)$ can be ex-

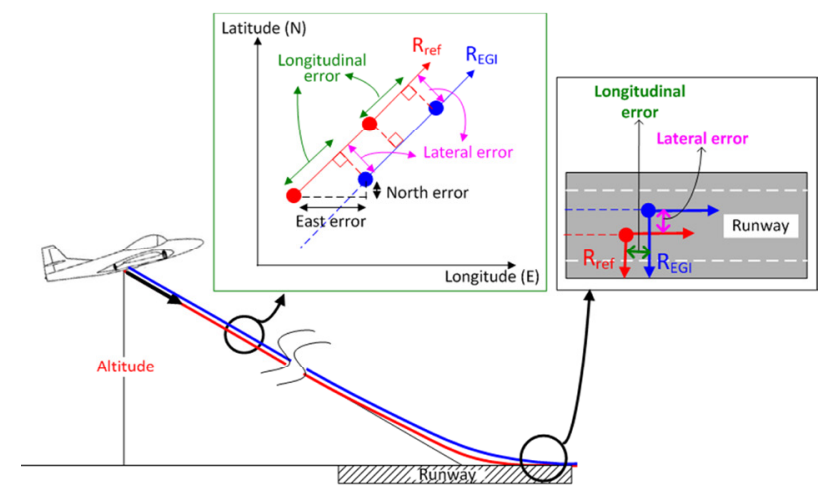

Fig. 5. North/east error vs longitudinal/lateral errors on a glide path.

pressed as Eq. (1) (Cai et al. 2011, Titterton \& Weston 2004). Thus, the coordinate transformation matrix for transforming the $\mathrm{n}$-frame into the $\mathrm{LLV}$-frame $\left(\mathrm{C}_{\mathrm{N}}^{\mathrm{L}}\right)$ can be expressed as Eq. (2). $C_{\theta}$ represents $\cos \theta$, and $S_{\theta}$ represents $\sin \theta$. Also, in the case of the coordinate transformation matrix for transforming the b-frame into the $n$-frame, the transposed matrix of Eq. (1) is used (Cai et al. 2011). Thus, the coordinate transformation matrix for transforming the $b$-frame into the LLVframe $\left(\mathrm{C}_{\mathrm{B}}^{\mathrm{L}}\right)$ can be expressed as Eq. (3).

$$
\begin{aligned}
\mathrm{C}_{\mathrm{N}}^{\mathrm{B}} & =\mathrm{C}_{\varphi} \cdot \mathrm{C}_{\theta} \cdot \mathrm{C}_{\psi} \\
& =\left[\begin{array}{lll}
\mathrm{c}_{11} & \mathrm{c}_{12} & \mathrm{c}_{13} \\
\mathrm{c}_{21} & \mathrm{c}_{22} & \mathrm{c}_{23} \\
\mathrm{c}_{31} & \mathrm{c}_{32} & \mathrm{c}_{33}
\end{array}\right] \\
& =\left[\begin{array}{ccc}
\mathrm{c}_{\theta} \mathrm{c}_{\psi} & \mathrm{c}_{\theta} \mathrm{s}_{\psi} & -\mathrm{s}_{\theta} \\
-\mathrm{c}_{\varphi} \mathrm{s}_{\psi}+\mathrm{s}_{\varphi} \mathrm{s}_{\theta} \mathrm{c}_{\psi} & \mathrm{c}_{\varphi} \mathrm{c}_{\psi}+\mathrm{s}_{\varphi} \mathrm{s}_{\theta} \mathrm{s}_{\psi} & \mathrm{s}_{\varphi} \mathrm{c}_{\theta} \\
\mathrm{s}_{\varphi} \mathrm{s}_{\psi}+\mathrm{c}_{\varphi} \mathrm{s}_{\theta} \mathrm{c}_{\psi} & -\mathrm{s}_{\varphi} \mathrm{c}_{\psi}+\mathrm{c}_{\varphi} \mathrm{s}_{\theta} \mathrm{s}_{\psi} & \mathrm{c}_{\varphi} \mathrm{c}_{\theta}
\end{array}\right] \\
\mathrm{C}_{\mathrm{N}}^{\mathrm{L}} & =\mathrm{C}_{\psi} \\
\mathrm{C}_{\mathrm{B}}^{\mathrm{L}} & =\left(\mathrm{C}_{\mathrm{L}}^{\mathrm{B}}\right)^{\mathrm{T}}=\left(\mathrm{C}_{\varphi} \cdot \mathrm{C}_{\theta}\right)^{\mathrm{T}} \\
& =\left[\begin{array}{ccc}
\mathrm{c}_{\theta} & \mathrm{s}_{\varphi} \mathrm{s}_{\theta} & \mathrm{c}_{\varphi} \mathrm{s}_{\theta} \\
0 & \mathrm{c}_{\varphi} & -\mathrm{s}_{\varphi} \\
-\mathrm{s}_{\theta} & \mathrm{s}_{\varphi} \mathrm{c}_{\theta} & \mathrm{c}_{\varphi} \mathrm{c}_{\theta}
\end{array}\right]
\end{aligned}
$$

where,

$$
C_{\psi}=\left[\begin{array}{ccc}
c_{\psi} & s_{\psi} & 0 \\
-s_{\psi} & c_{\psi} & 0 \\
0 & 0 & 1
\end{array}\right]
$$




$$
\begin{aligned}
\mathrm{C}_{\theta} & =\left[\begin{array}{ccc}
\mathrm{c}_{\theta} & 0 & -\mathrm{s}_{\theta} \\
0 & 1 & 0 \\
\mathrm{~s}_{\theta} & 0 & \mathrm{c}_{\theta}
\end{array}\right] \\
\mathrm{C}_{\varphi} & =\left[\begin{array}{ccc}
1 & 0 & 0 \\
0 & \mathrm{c}_{\varphi} & \mathrm{s}_{\varphi} \\
0 & -\mathrm{s}_{\varphi} & \mathrm{c}_{\varphi}
\end{array}\right]
\end{aligned}
$$

The position error estimated from the result of the navigation system and the reference trajectory can be expressed as Eq. (4). The position that the navigation system outputs $\left(\widehat{\mathrm{P}}_{\mathrm{EGI}}\right)$ and the position that the reference trajectory outputs $\left(\widehat{\mathrm{P}}_{\mathrm{REF}}\right)$ are different as they are the center of gravity of the air vehicle $\left(\mathrm{P}_{\mathrm{CG}}\right)$ and the position of the GPS antenna $\left(\mathrm{P}_{\mathrm{GPS} \text { Ant }}\right)$, respectively. Thus, when the NED errors are calculated by correcting the distance (i.e., lever arm), it can be expressed as Eq. (5) (Park \& Shin 2012).

$$
\begin{aligned}
& \widehat{\mathrm{e}}_{\mathrm{G}}=\left(\widehat{\mathrm{P}}_{\mathrm{REF}}-\widehat{\mathrm{P}}_{\mathrm{EGI}}\right)_{\mathrm{G}} \\
& \mathrm{de}_{\mathrm{N}}=\mathrm{d} \hat{\mathrm{e}}_{\mathrm{N}}+\mathrm{L}_{\mathrm{N}}
\end{aligned}
$$

where,

$$
\begin{aligned}
& \widehat{\mathrm{P}}_{\mathrm{EGI}}=\left(\mathrm{P}_{\mathrm{CG}}+\mathrm{e}_{\mathrm{EGI}}\right)_{\mathrm{G}} \\
& \widehat{\mathrm{P}}_{\mathrm{REF}}=\left(\mathrm{P}_{\mathrm{GPS} A n t}+\mathrm{e}_{\mathrm{REF}}\right)_{\mathrm{G}} \\
& \mathrm{L}_{\mathrm{N}}=\left(\mathrm{C}_{\mathrm{N}}^{\mathrm{B}}\right)^{-1} \mathrm{~L}_{\mathrm{B}}=\mathrm{C}_{\mathrm{B}}^{\mathrm{N}} \mathrm{L}_{\mathrm{B}} \\
& \mathrm{L}_{\mathrm{B}}=\left(\mathrm{P}_{\mathrm{CG}}-\mathrm{P}_{\mathrm{GPS} \text { Ant }}\right)_{\mathrm{B}} \\
& \mathrm{de}_{\mathrm{N}}=\left[\begin{array}{l}
\mathrm{dN} \\
\mathrm{dE} \\
\mathrm{dD}
\end{array}\right]=\left[\begin{array}{c}
\frac{\mathrm{d} \mu}{\operatorname{atan}\left(\frac{1}{\mathrm{R}_{\mathrm{M}}}\right)} \\
\frac{\mathrm{dl}}{\operatorname{atan}\left(\frac{1}{\mathrm{R}_{\mathrm{N}} \cos \mu_{0}}\right)} \\
-\mathrm{dh}
\end{array}\right] \\
& \mathrm{R}_{\mathrm{N}}=\frac{\mathrm{R}}{\sqrt{1-\left(2 \mathrm{f}-\mathrm{f}^{2}\right) \text { si }^{2} \mu_{0}}} \\
& R_{M}=R_{N} \frac{1-\left(2 f-f^{2}\right)}{\sqrt{1-\left(2 f-f^{2}\right) \text { si }^{2} \mu_{0}}} \\
& \mathrm{~d} \mu=\mu-\mu_{0} \text { : latitude difference } \\
& \mathrm{dl}=\mathrm{l}-\mathrm{l}_{0} \quad \text { : longitude difference } \\
& \mathrm{dh}=\mathrm{h}-\mathrm{h}_{0} \text { : height difference } \\
& 1 / \mathrm{f}=298.257223563 \\
& \mathrm{R}=6378137 \mathrm{~m}
\end{aligned}
$$

( ) $)_{\mathrm{G}}$ represents the geodetic frame, ( ) ${ }_{\mathrm{B}}$ represents the body frame, and ()$_{\mathrm{N}}$ represents the navigation frame. $\mathrm{e}_{\mathrm{EGI}}$ and $\mathrm{e}_{\mathrm{REF}}$ represent the actual errors of the navigation system and the reference trajectory, respectively. Using the NED errors, the LLV errors can be expressed as Eq. (6), with the use of Eqs. (2) and (4).

$$
\begin{aligned}
\operatorname{de}_{\mathrm{L}} & =\mathrm{C}_{\mathrm{N}}^{\mathrm{L}} \mathrm{de}_{\mathrm{N}} \\
& =\left[\begin{array}{ccc}
\mathrm{c}_{\psi} & \mathrm{s}_{\psi} & 0 \\
-\mathrm{s}_{\psi} & c_{\psi} & 0 \\
0 & 0 & 1
\end{array}\right]\left(\mathrm{d} \hat{\mathrm{e}}_{\mathrm{N}}+\mathrm{L}_{\mathrm{N}}\right)
\end{aligned}
$$

\section{FLIGHT TEST RESULTS}

\subsection{Flight Test Environment and Test Procedure}

An actual flight test was performed by loading a DGPS/ INS system, which uses the Differential-GPS (DGPS) technique that provides a position accuracy of several meters (Farrell 2000, Redmill et al. 2001), onto an air vehicle, and the longitudinal/lateral errors were analyzed. As for the reference equipment for evaluating the performance of the DGPS/INS system, DL-V3 (NovAtel Inc.), which is high-precision navigation equipment that can generate carrier phase DGPS (CDGPS) reference trajectories with a precision of $5 \mathrm{~mm}$, was used. As the air vehicle used in the test landed on a runway, spaces were sufficiently secured in the heading direction (i.e., longitudinal axis). Therefore, in this test, whether the lateral error is within the range in which the air vehicle can safely land within the width of the runway was examined.

Fig. 6 shows the block diagram of the flight test equipment. Inside the air vehicle, the DGPS/INS system, which is the performance evaluation target, and the DL$\mathrm{V} 3$, which is the rover station (RS) for reference trajectory generation, were installed. At the control tower outside the air vehicle, the base station (BS) was installed. The GPS antenna was shared by the DGPS/INS system and the rover station DL-V3, using a GPS signal distributor. The rover station DL-V3 was installed at the same space where the DGPS/INS system was installed, using a jig.

During the inspection of the air vehicle before performing the flight test, the self-inspection and alignment of the DGPS/INS system were conducted. For the alignment, self-alignment was conducted using a gyroscope and an

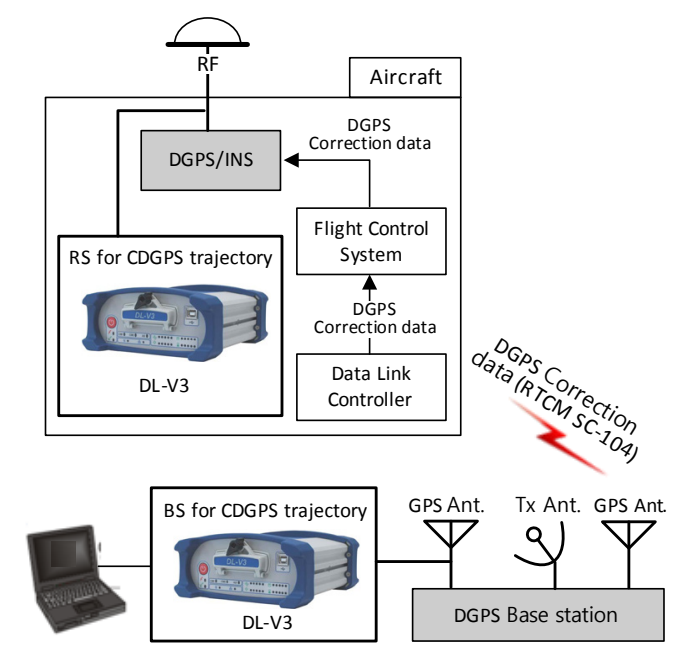

Fig. 6. Block diagram of the flight test equipment. 


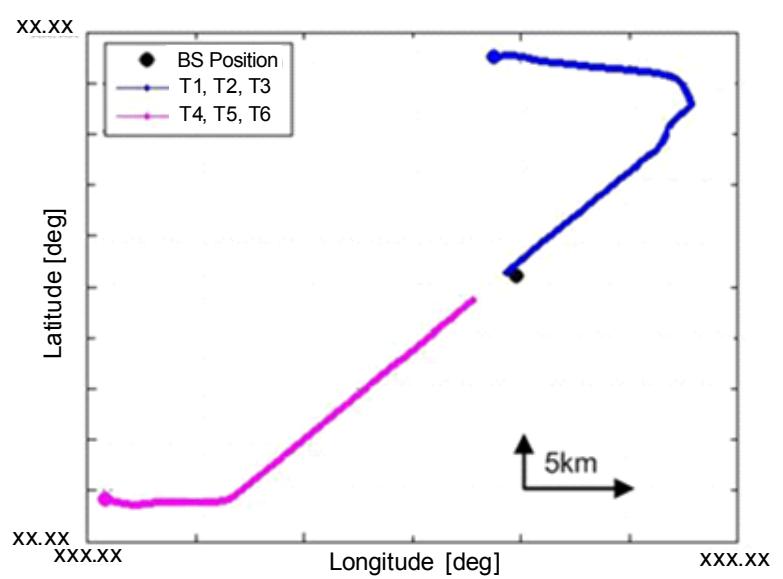

Fig. 7. Flight trajectories.

accelerometer, and after the completion, the flight test was performed. During the takeoff and landing, it was operated at the DGPS/INS mode by receiving the DGPS correction information (RTCM1). During the flight test, the DL-V3 installed in the air vehicle and on the ground received the GPS raw measurement data for post-processing CDGPS reference trajectory generation. In this regard, the base station on the ground was installed at the spot where a geodetic survey had been performed in advance.

After the flight test, for the performance evaluation of the DGPS/INS system, the north/east error analysis, which is the existing method, and the longitudinal/lateral error analysis, which is the newly proposed method, were performed. For the performance analysis, the GPS raw measurements obtained from the rover station DL-V3 installed in the air vehicle and from the base station DLV3 were extracted, and the CDGPS reference trajectory was generated using Grafnav, which is the commercial CDGPS post-processing software from NovAtel Inc. As the output position of the DGPS/INS system is the center of
Table 1. Distance between the DGPS base station and the air vehicle $(\mathrm{km})$.

\begin{tabular}{rccc}
\hline Test & Horizontal distance & Vertical distance & 3D \\
\hline T1 3 & 24 & 1 & 24 \\
T4 6 & 30 & 1 & 30 \\
\hline
\end{tabular}

gravity of the air vehicle, it is not consistent with the CDGPS reference trajectory. Thus, if the north/east errors between the DGPS/INS system and the obtained CDGPS reference trajectory are calculated, these north/east errors include the GPS lever arm error. Therefore, the output positions of the CDGPS reference trajectory and the DGPS/INS system were matched by performing the GPS lever arm correction using the attitude data obtained from the DGPS/INS system. Then the NED errors and the longitudinal/lateral errors were calculated and analyzed using the equations described in Section 2.2.

Flight tests were performed a total of six times, and for each test, the DGPS/INS system setting and the air vehicle operation environment were the same. However, each test was performed on a different date, and the trajectories of T1 3 and T4 6 were different, as shown in Fig. 7. Thus, the GPS satellites used in each test and the arrangement of the satellites could be different. The landing sections (T1 T6) for the six flight tests, in which the DGPS/INS navigation was performed, were from the point, at which the distance between the DGPS base station and the air vehicle was about $24 \mathrm{~km} \sim 30 \mathrm{~km}$, to the landing point, as summarized in Table 1.

\subsection{Flight Test Results}

Figs. 8 and 9 show the north/east errors and longitudinal/ lateral errors for T1 T4 and T5 T6, respectively. Table 2 summarizes the root-mean-square (RMS) values of the north/east errors and longitudinal/lateral errors for each
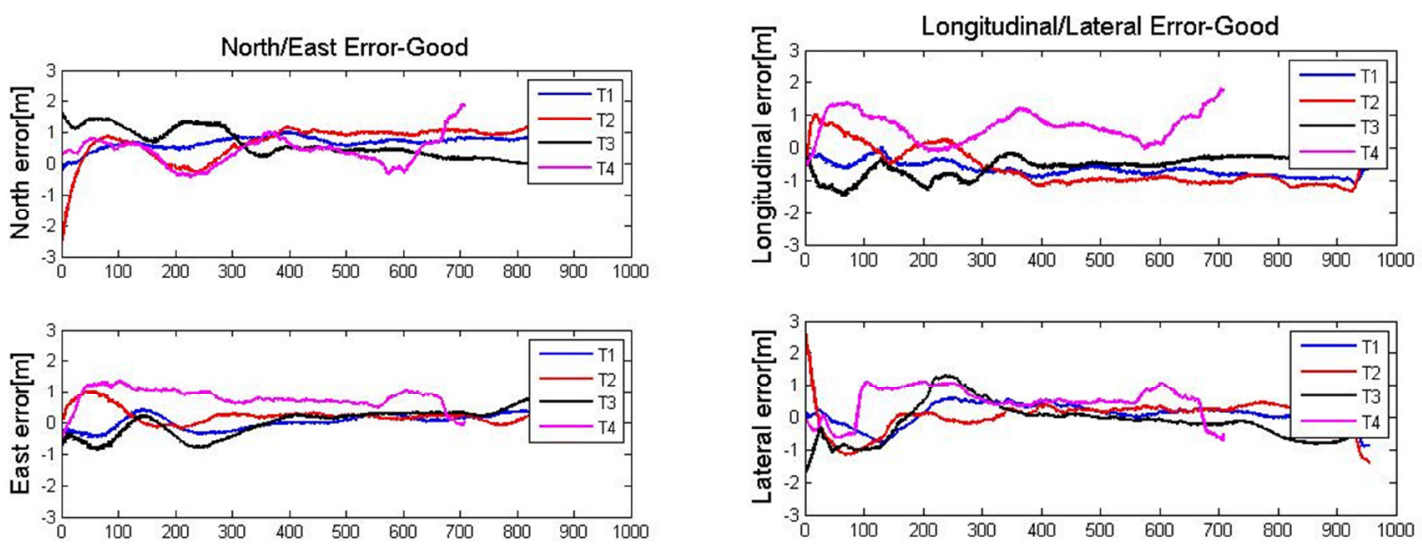

Fig. 8. North/east and longitudinal/lateral position errors with good DGPS correction. 

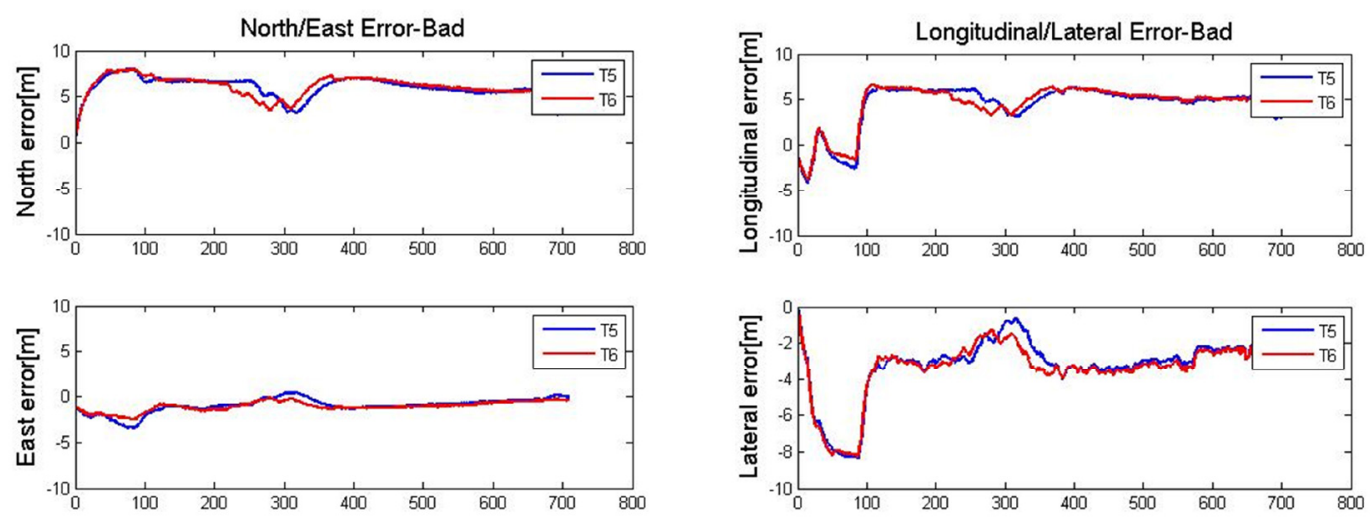

Fig. 9. North/east and longitudinal/lateral position errors with bad DGPS correction.

test. As shown in Fig. 8, the results of the normal navigation performance including the DGPS correction indicated that for T1 T4, the north/east errors and the longitudinal/lateral errors were $1 \mathrm{~m}$ RMS, and were within $\pm 2 \mathrm{~m}$ in the test section. Therefore, it was found that if the DGPS correction is normally performed, the auto-landing of the air vehicle on the runway is possible.

As shown in Fig. 9, for T5 T6, the north error was $6 \mathrm{~m}$ RMS, and an error of 6 8 m occurred almost consistently in the test section. Thus, the required navigation performance could not be satisfied. The cause of this error was found to be the increase in the tropospheric error due to the strict DGPS correction data integrity constraints of the GPS receiver and the low elevation angle of the GPS satellite data included in the DGPS correction data. If the DGPS correction data integrity constraints are strict, the DGPS correction data, which can be sufficiently used, may not be used. As shown in Fig. 10, when the altitude difference between the air vehicle and the DGPS base station is about $1 \mathrm{~km}$ or more, the tropospheric error increases as the elevation angle of the GPS satellite included in the DGPS correction data decreases. However, the longitudinal/lateral errors were $5 \mathrm{~m}$ RMS and $3.8 \mathrm{~m}$ RMS, respectively. In the case of the lateral error, the error increased from the point at which the DGPS/INS navigation started. Then it decreased to less than $4 \mathrm{~m}$ after about 100 seconds, and decreased to less than $2 \mathrm{~m}, 200$ seconds before the landing. Therefore, it was found that the auto-landing of the air vehicle on

Table 2. North/east and longitudinal/lateral position errors (RMS).

\begin{tabular}{c|c|c|c|c}
\hline \multirow{2}{*}{ Test } & \multicolumn{2}{|c|}{ North/East errors } & \multicolumn{2}{c}{ Longitudinal/Lateral errors } \\
\cline { 2 - 5 } & North error & East error & Longitudinal error & Lateral error \\
\hline T1 & 0.75 & 0.22 & 0.63 & 0.36 \\
T2 & 0.91 & 0.33 & 0.59 & 0.47 \\
T3 & 0.73 & 0.45 & 0.63 & 0.59 \\
T4 & 0.61 & 0.86 & 0.80 & 0.68 \\
T5 & 6.07 & 1.27 & 5.03 & 3.63 \\
T6 & 6.16 & 1.19 & 5.03 & 3.75 \\
\hline
\end{tabular}

the runway is possible because the lateral error decreased sufficiently although the north/east errors did not satisfy the required performance.

\section{CONCLUSIONS}

In this study, a method for analyzing the longitudinal/ lateral errors of a navigation system was newly proposed as the navigation performance test method for evaluating the safety during the auto-landing of an air vehicle. Also, flight tests were performed six times, and the north/east errors, which is the existing method, was analyzed and compared with the proposed method. Then the safety of auto-landing was examined based on the results of the performance analysis. As the air vehicle used in the flight test landed on a runway, spaces were sufficiently secured in the direction of the longitudinal axis. Therefore, whether the lateral error is within the range in which the air vehicle can safely land within the width of the runway was examined. As for $\mathrm{T} 1 \sim \mathrm{T} 4$, which showed normal navigation performance in the test, the auto-landing of the air vehicle was found to be possible because all the errors were within $2 \mathrm{~m}$ in the landing section. As for T5 T6, the auto-landing of the air

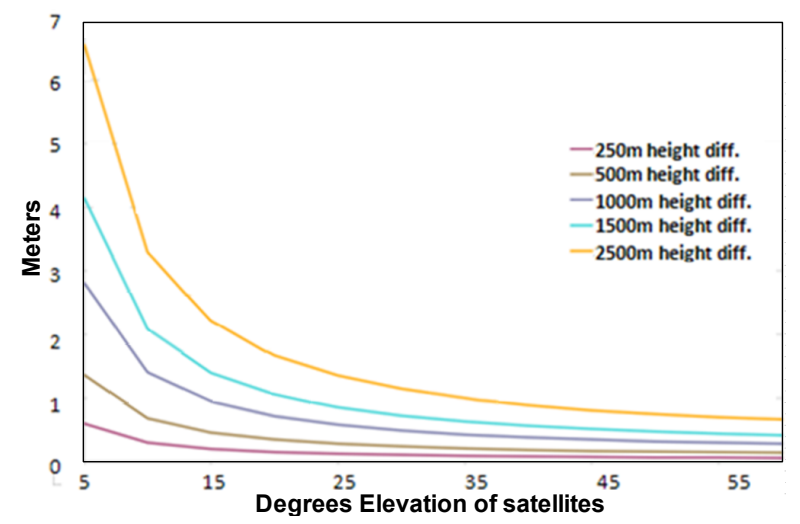

Fig. 10. Tropospheric error for height difference and satellite elevation angles. 
vehicle was also found to be possible because the lateral error decreased to less than $2 \mathrm{~m}, 200$ seconds before the landing although the north error did not satisfy the required navigation performance. Therefore, it was found that during the navigation performance evaluation of an air vehicle for auto-landing, the longitudinal/lateral errors as well as the north/east errors need to be examined.

\section{REFERENCES}

Boeing 2009, Statistical Summary of Commercial Jet Airplane Accidents: Worldwide Operations 1959-2008 (Washinton: Boeing Commercial Airplanes), 10-22. http://www.airsafe.com/events/models/statsum2008. pdf

Cai, G., Chen, B. M., \& Lee, T. H. 2011, in Advanced in Industrial Control, Unmanned Rotorcraft Systems (New York: Springer), 23-34. http://dx.doi.org/ 10.1007/9780-85729-635-1

Farrell, J. 2000, Differential GPS Reference Station Algorithm-Design and Analysis, IEEE Transactions on Control Systems Technology, 8, 519-531

Farrell, J. \& Barth, M. 1998, The Global Positioning System and Inertial Navigation (New York: McGraw-Hill), 4

Noh, K. M. \& Agarwal, R. K. 2000, Automatic Landing System Design Using Fuzzy Logic, Journal of Guidance, Control, and Dynamics, 23, 298-304

Office of the Secretary of Defense 2005, Unmanned Aircraft Systems (UAS) Road map 2005-2030, Office of the Secretary of Defense, H-4-5

Park, J. H. \& Shin, D. H. 2012, Comparison Between DCM and Quaternion Transformation in Lever Arm Compensation of Reference System for Flight Performance Evaluation of DGPS/INS, JKGS, 1, 45-49. http://dx.doi.org/ 10.11003/JKGS.2012.1.1.045

Redmill, K. A., Kitajima, T., \& Ozquner, U. 2001, DGPS/ INS Integrated Positioning for Control of Automated Vehicle, IEEE Intelligent Transportation Systems Conference Proceeding, 172-178

Sabatini, R. \& Palmerini, G. B. 2000, Differential Global Positioning System (DGPS) for Flight Test, NATO Research and Technology Organization, Flight Test instrumentation Series, 21, 6-1-6-7

Shue, S. P. \& Agarwal, R. K. 1999, Design of Automatic Landing Systems Using Mixed $\mathrm{H} 2 / \mathrm{H} \infty$ Control, Journal of Guidance, Control and Dynamics, 22, 103-114

Titterton, D. \& Weston, J. 2004, Strapdown Inertial Navigation Technology, 2nd ed. (London: The Institution of Engineering and Technology), 36-46

Yoo, K. J, Park, H. W., \& Kim, Y. H. 2011, Radar Altimeter Measurement Error Analysis According to Terrain and Antenna Beam Width, KSAS Spring Conference, 190193
Yoon, J. S., Kwak, H. J., Kim, Y. H., Shin, Y. J., Yoo K. J., \& Yu, M. J. 2013, The Performance Analysis of an Airborne Radar Altimeter based on Simultaneously Acquired LiDAR Data, Korean Journal of Remote Sensing, 29, 81-94

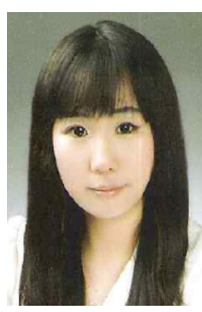

Ji Hee Park received the B.S and M.S. degree in Information Communication from Chungnam National University, Korea, in 2008 and 2010, respectively. She is currently working as a researcher in Avionics R\&D lab of LIGNexl. Her research interests include GPS/INS and satellite navigation.

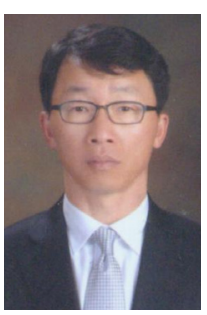

Hong Sik Park received the B.S degree in Aeronautics from Air force Academy, Korea, in 1988. He is currently working as a research engineer in Avionics R\&D lab of LIGNex1. His research interests include avionics system of fixed and rotary wing.

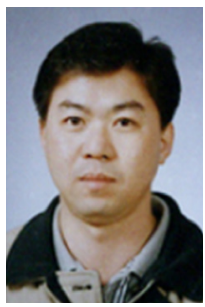

Chol Soo Shin received the B.S and M.S degree in electronic engineering from Myongji University, Korea, in 1990 and 1992, respectively. He is currently working as a project team leader in Avionics R\&D lab of LIGNex1. His research interests include avionics system of fixed and rotary wing.

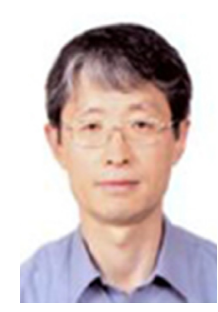

Young-Wo Jo received the B.S degree in electric engineering from Seoul National University, Korea, in 1986 and M.S degree in computer science from KAIST 1995, respectively. He is currently working as a project team leader in Avionics Department, 7th Division, of ADD. His research interests include avionics system of fixed and rotary wing.

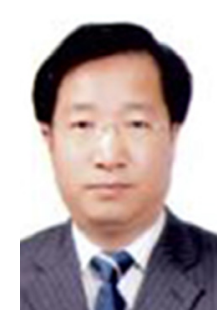

Dong-Ho Shin received the B.S and M.S. degree in Electronics from Kyungpook National University, Korea, in 1989 and 1993, respectively. He also received Ph. D. degree in Electrics from Chungnam National University, Korea in 2002. He is currently working as a principal researcher in Avionics Department, 7th Division, of ADD. His research interests include GPS/INS, Aircraft navigation system and satellite navigation. 